\title{
Enrichment of Copper, Lead, and Tin by Mechanical Dry Processing of Obsolete Printed Circuit Board Residues
}

\author{
Marcelle de Fátima da Silva ${ }^{a}$, Achilles Junqueira Bourdot Dutra ${ }^{a}$, Marcelo Borges Mansur ${ }^{a} * \mathbb{C}$ \\ aPrograma de Pós-Graduação em Engenharia Metalúrgica e de Materiais (COPPE), Universidade \\ Federal do Rio de Janeiro (UFRJ), Rio de Janeiro, RJ, Brasil
}

Received: May 16, 2019; Revised: July 23, 2019; Accepted: August 27, 2019

\begin{abstract}
Waste printed circuit boards (WPCB) residues were mechanically processed to concentrate their metal content aiming to reduce costs of the subsequent recovery of copper, tin, and lead. A fully dry route was proposed to avoid the generation of liquid effluents that would require additional treatment. Firstly, $10.7 \%$ of the residue was segregated by magnetic separation; the remaining nonmagnetic fraction was comminuted and sieved. Ceramic and polymer materials ( $1 / 3$ of the total weight) concentrated in the finer and the coarser size fractions, while metallic materials ( $2 / 3$ of the total weight) concentrated in the intermediate size fraction (90.7, 94.5 , and $95.6 \%$ of the total copper, tin, and lead contained in the milled WPCB, respectively). The fractions between $0.3-1.20 \mathrm{~mm}$ were submitted to gravity separation using a zig-zag air classifier; enrichment of copper (from $43 \pm 11 \%$ to $68 \pm 5 \%$ ), tin (from $10 \pm 3 \%$ to $17 \pm 1 \%$ ), and lead (from $4 \pm 1 \%$ to $6.4 \pm 0.5 \%$ ) were obtained.
\end{abstract}

Keywords: printed circuit boards, zig-zag air classifier, mechanical processing.

\section{Introduction}

The increase in demand for electrical and electronic equipment and shorter life span brings to a global sustainability concern. Waste electrical and electronic equipment (WEEE) constitute urban mineral resources for recovery of different metals. Their disposal as open-pit waste, incineration and/ or landfill generates hazardous by-products and gaseous emissions, which impacts the environment, human health and the sustainable economy. The core part of WEEE constitute the waste printed circuit board (WPCB), which stands out as one of the most difficult component to treat and most valuable part to recycle ${ }^{1-2}$. They contain approximately $23 \%$ of polymers, $38 \%$ of metals, and $49 \%$ of ceramic materials, however, such composition change considerably depending on the electronic device type, model, year of fabrication, $\operatorname{etc}^{3-4}$. The main metals present in WPCB include copper, lead, and tin. Other elements can also be found in relatively lower contents as is the case of iron, nickel, zinc, as well as precious metals like gold, silver, and palladium.

Different strategies based on reuse, recovery and recycling of WPCB are under development worldwide. Pyrometallurgy, hydrometallurgy, biometallurgy, or hybrid processing routes recycling techniques have been proposed to recover their valuable components ${ }^{5-10}$. Such treatments may contribute to reduce the environmental impacts caused by the extraction of high-valued materials as well as to eliminate the discharge of highly toxic materials from nature. As generally seen in WPCB, the content of some metals may surpass the typical values found in their respective ores ${ }^{1-2}$. More efficient routes

*e-mail: marcelo.mansur@metalmat.ufrj.br normally include mechanical processing of WPCB with the first phase aiming to reduce the volume of material to be treated, however effluents and residues can be generated thus requiring adequate environmental conditioning. Even those, all routes present technical limitations. For instance, the formation of volatile organic compounds like dioxins during smelting operations of WPCB will require off-gas treatment equipment to avoid toxic gas emissions in pyrometallurgical processing, the handling and disposal of strongly acidic solutions must be attempted in hydrometallurgical operations and the time required for decomposition in biological routes must be considered. In fact, the key of a valuable metal recovery process relies on a robust separation technique that can separate different metal components from a complicated metal bearing mixture in a low cost operation and without producing further negative impacts to environment ${ }^{11}$.

Independently of the chosen route, the metal recovery efficiency may increase if WPCB are preprocessed by physical methods, which are based on differences of magnetic, density, or size properties, aiming at concentrating the metal fraction through its segregation from the polymer and ceramic fractions ${ }^{12-16}$. For instance, He and Duan ${ }^{17}$ verified that metal components in WPCB are mainly distributed in sieving products with larger fractions; in the analyzed samples, the optimum size fraction to recover the metals from WPCB was $0.074-0.250 \mathrm{~mm}$, which ensured metal concentration recovery efficiency greater than $84 \%$ using reverse flotation. Zhang et al. ${ }^{18}$ included mechanical processing (comminution, sieving, and magnetic separation) as pretreatment of WPCB before triboelectric separation; the recovery of the nonmetallic products increased from $24.88 \%$ to $35.36 \%$. Also, in the study of Xia et al. ${ }^{19}$, the WPCB was first cut into pieces 
$(<0.40 \mathrm{~mm})$, comminuted in a vibratory mill (sizes between 74-1000 $\mu \mathrm{m}$ ), homogenized, and then separated into two fractions using a shaking table, which were submitted to a subsequent hybrid treatment: the metal rich fraction was treated by pyrometallurgical methods, whereas the metal poor fraction was submitted to bio-leaching where $85.23 \%$ zinc, $76.59 \%$ copper, and $70.16 \%$ aluminum were recovered. Zhang et al..$^{20}$ used grinding, magnetic separation, milling, and sieving to treat WPCB. The processed material was submitted to an electrostatic corona separation to obtain metal and nonmetal fractions. The residual metals present in the nonmetallic fraction were processed in a fluidized bed. The metals recovery in the size fractions of $1.0-0.5 \mathrm{~mm}$, $0.50-0.25 \mathrm{~mm}$, and $<0.25 \mathrm{~mm}$ were $86.39 \%, 82.22 \%$, and $76.63 \%$, respectively.

In the present study, the mechanical processing methods commonly used in ore treatment were applied to separate non-metallic (plastic and ceramics) and metallic fractions of WPCB, aiming to concentrate copper, lead, and tin in the metallic fraction. The proposed route included comminution and magnetic separation, followed by a zig-zag air classifier; therefore no water was required to separate the metallic and non-metallic fractions of the residue. The advantage of the fully dry route proposed in the present work is to avoid the generation of liquid effluent streams that would need to be adequately treated, besides providing a very fast separation if compared to other physical separation techniques.

\section{Experimental}

\subsection{Collection and manual dismantling}

In order to develop a route to process different types of printed circuit boards, WPCB from obsolete computers of distinct types, models, and fabrication years were collected at Federal University of Rio de Janeiro campus. The material was manually dismantled to remove capacitors, resistors, CPU (central processing unit), fans, heat-dissipators, and other soldered components that could bend during comminution and reduce the liberation efficiency of metal, ceramic, and polymer fractions. In addition, components containing precious metals like gold, silver and palladium were separated to specific treatment ${ }^{21}$. After manual dismantling, approximately 3.6 $\mathrm{kg}$ of WPCB were obtained, thus corresponding to $86.5 \%$ of the total mass, which was subjected to the subsequent mechanical processing.

\subsection{Comminution}

Firstly, the WPCB were cut into small pieces (below 10 $\mathrm{cm}$ length and $5 \mathrm{~cm}$ width) using a laboratory-scale shredder (Fragmaq, FT/75 model, $7.5 \mathrm{hp}$ ). Then, milling of the cut material was run using a Wyllie knife mill (CIENLAB, CE-430 model, $2 \mathrm{hp}$, exit opening $2.5 \mathrm{~mm}$ ).

\subsection{Magnetic separation}

A laboratory-scale magnetic separator designed at UFRJ with a $3000 \mathrm{G}$ magnetic field produced by a rare earth magnet was used. The operation was performed under dry conditions (17 $\mathrm{rpm}$ rotation, $80 \mathrm{~g} / \mathrm{min}$ feed rate). The material was fed to the conveyor belt of the equipment, being separated into two fractions: the magnetic fraction retained by the magnetic field and the nonmagnetic fraction, which was submitted to the subsequent mechanical processing step to concentrate copper, tin, and lead.

\subsection{Sieving}

The nonmagnetic fraction was screened by sieves with openings of $2,1.2,0.85,0.71,0.5,0.3$, and $0.125 \mathrm{~mm}$ (-9\# to $+115 \#$ mesh Tyler) and bottom. The sample was placed at the top sieve and the set of sieves was shaken for $15 \mathrm{~min}$, which was found adequate to provide complete separation for the sieve shaker used (Ro-tap). The fractions from each sieve were weighed and submitted to the gravity pneumatic separation step; samples ( $1 \mathrm{~g}$ ) were withdrawn for chemical analysis.

\subsection{Zig-zag pneumatic separation}

The pneumatic separation tests were performed using a laboratory-scale zig-zag air classifier, aiming to concentrate the metallic content of the nonmagnetic fraction of each size fraction obtained in the sieving step. The advantage of using an air classifier is to avoid the generation of liquid effluents in the mechanical processing of WPCB. In addition, given the equipment has no internal parts, it quickly performs metal separation requiring very low energy consumption. The operation of the zig-zag air classifier is schematically shown in Figure 1. For each test, samples of approximately $10 \mathrm{~g}$ of the waste were fed into the classifier. The upward air flowing in a counter current mode was provided by a blower (B-air Blowers, KP-1200 model, maximum flow rate $=18.5 \mathrm{~m}^{3} / \mathrm{h}$ ) which immediately drags the light fraction to the upper direction, while the heavy fraction is directed by gravity to the bottom exit of the classifier. Samples ( $1 \mathrm{~g})$ of both fractions were withdrawn for chemical analysis. The air flow rate was defined using Schytil's diagrams.

In the zig-zag air classifier, particles are classified based on their falling behavior in the channel in which air stream flows upward. To determine the occurrence of fluidization in the equipment for a given operating condition, the Schytil's diagram for gas/solid suspensions was plotted for each particle size and particles desity. This diagram is, a $\log -\log$ plot of the dimensionless numbers Froude $\left(\mathrm{Fr}=\mathrm{v}^{2}\right.$ / gd) versus Reynolds $(\mathrm{Re}=\mathrm{Re}=\mathrm{rvd} / \mu)$, assuming constant velocity (Eq. 1) and constant particle diameter (Eq. 2) as given by $\mathrm{Pacheco}^{22}$ :

$$
\log F r=-\log \operatorname{Re}+\log \left(\frac{v^{3} \rho}{\mu g}\right)
$$




$$
\log F r=-2 \log \mathrm{Re}+\log \left(\frac{\mu^{2}}{\rho^{2} g d^{3}}\right)
$$

which are obtained by substituting both dimensionless number definitions and expliciting the particle diameter (d) or the velocity (v), respectively. In the equations, $\rho$ and $\mu$ are density and viscosity of fluid, respectively, and $g$ is the acceleration due to gravity. Despite not considering the particle shape, the Schytil's diagram represents the conditions

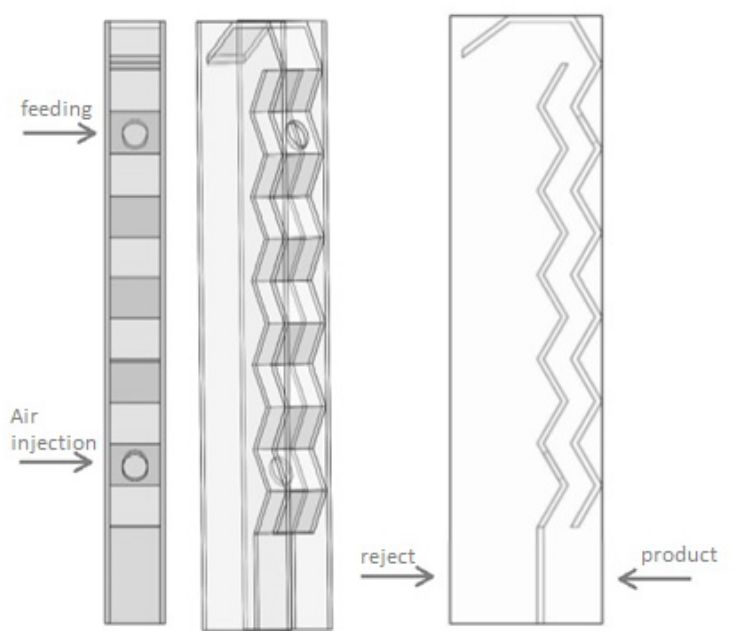

Figure 1. Operation scheme of the zig-zag air classifier.

that will result in a fluidized bed rather than a fixed bed remaining or going pneumatic transport.

From the Ergun equation, used to calculate the pressure drop across a randomly packed bed of spherical particles for laminar and turbulent flow conditions, equations determining the domain between fixed and fluidizing beds (Eq. 3), and the domain between fluidizing bed and pneumatic transport (Eq. 4) can be derived ${ }^{23}$ :

$$
\begin{gathered}
F r=\frac{\varepsilon^{3}\left(\rho_{s}-\rho\right)}{\rho\left[\frac{150(1-\varepsilon)}{\operatorname{Re}}+1.75\right]} \\
F r=\frac{4\left(\rho_{s}-\rho\right) \varepsilon^{4,65}}{3 C_{d} \rho_{s}}
\end{gathered}
$$

where $\rho_{\mathrm{s}}$ is the solid density, $\varepsilon$ is the void fraction, and $\mathrm{C}_{\mathrm{d}}$ is the drag coefficient whose value depends on Reynolds number.

\subsection{Characterization of the solid fractions}

Samples of the material were withdrawn along the treatment route for physical and chemical characterization.
The relative density of the samples for each fraction size after sieving was determined using the picnometric method. Scanning electron microscopy (SEM) images were obtained to analyze the morphology structure of the samples using a JEOL JSM-6460 LV equipment, which was equipped with an energy dispersive spectrometer (EDS) to obtain a semiquantitative elemental composition of the samples. Eventually, given the heterogeneity of the samples, the content of copper, lead, and tin of each size fraction after sieving step was determined using an atomic absorption spectrophotometer (Shimadzu, AA 6800 model) after previous digestion of the samples $(1 \mathrm{~g})$ in $20 \mathrm{~mL}$ of aqua regia $\left(3 \mathrm{HCl}: 1 \mathrm{HNO}_{3}\right.$, both reagents analytical grade, Vetec).

\section{Results and Discussion}

\subsection{Comminution, magnetic separation, and sieving steps}

Approximately, $3.6 \mathrm{~kg}$ of WPCB were obtained after manual dismantling, being first subjected to the comminution step using a shredder and a knife mill, followed by magnetic separation, and then by sieving steps.

Table 1 summarizes the total mass balances of these steps. The mass loss in the comminution step was negligible, below $1 \%$. This step was relatively easy to perform because the collected material was previously dismantled; in fact, the components that could bend during crushing and milling operations (capacitors, resistors, CPUs, fan/cooler, heatdissipator, and soldered components) were manually removed. Moreover, as the WPCB metal fraction is usually covered by ceramic and polymer materials ${ }^{5}$, the comminution step resulted in the liberation of such fractions. In particular, the mass difference $(10.7 \%)$ verified in the magnetic separation step corresponds to the magnetic fraction originally present in the WPCB, basically comprising electronic components that remain in the waste ${ }^{18}$. No mass loss was verified in the sieving step.

Table 1. Total mass balance of comminution, magnetic separation, and sieving steps.

\begin{tabular}{lcccc}
\hline Step & Input & Output & \multicolumn{2}{c}{ Mass difference } \\
& $(\mathrm{g})$ & $(\mathrm{g})$ & $\mathrm{g}$ & $\%$ \\
\hline Comminution & 3591 & 3571 & 20 & 0.6 \\
Magnetic separation & 3571 & 3188 & 383 & 10.7 \\
Sieving & 3188 & 3188 & 0 & 0.0 \\
\hline
\end{tabular}

The size distribution of the WPCB obtained in the sieving step is shown in Figure 2. Approximately $66 \%$ of the material concentrate in size fractions higher than 0.85 $\mathrm{mm}$, corroborating previous studies ${ }^{12,24}$, whereas less than $10 \%$ of the sample consists of fine material $(<125 \mathrm{~mm})$.

Samples of each size fraction were digested in aqua regia, resulting in two main fractions: $51 \%$ of soluble 


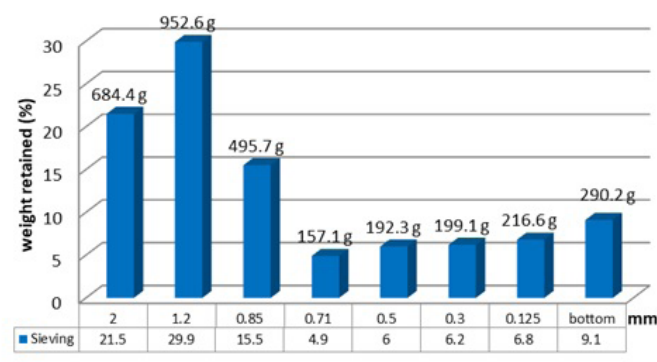

Figure 2. Size fractions distribution of WPCB after comminution and magnetic separation steps.

(predominance of metals - $36.9 \%$ copper, $6.6 \%$ tin, and $2.6 \%$ lead) and $49 \%$ of insoluble (predominance of polymers and ceramics) materials. Such figures are the average values calculated for all fractions studied. As shown in Figure 3 , the amount of soluble and insoluble materials changed with size particle. Soluble materials concentrate in some intermediate fractions $(0.71-2 \mathrm{~mm})$ and insoluble materials concentrate in the finest fractions $(<0.3 \mathrm{~mm})$, since ceramic and polymeric materials are relatively easier to be crushed, and in the coarsest fraction ( $>2 \mathrm{~mm}$ ), due to certain irregularity in size, whereas approximately the same content of soluble and insoluble materials was also verified in the remaining intermediate fractions $(0.3-0.71 \mathrm{~mm})$.

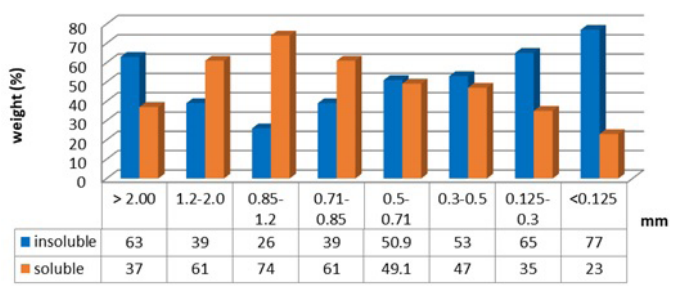

Figure 3. Distribution of soluble (metals) and insoluble (ceramic and polymer) materials in aqua regia with particle size fraction.

The distribution of copper, lead, and tin comprising the soluble fractions of WPCB for different particle sizes is shown in Figure 4. In terms of percentage, such metals concentrate mainly in the intermediate size fractions $(0.125-2 \mathrm{~mm})$. For copper, contents higher than $25 \%$ were obtained in this size fraction range, reaching $60.8 \%$ in the size fraction $0.85-1.2$ $\mathrm{mm}$. For tin, the average content was $10 \pm 3 \%$ in the size fraction $0.3-1.2 \mathrm{~mm}$, reaching $14.6 \%$ for the $0.85-1.2 \mathrm{~mm}$ size fraction. For lead, an average content of $3 \pm 1 \%$ was obtained in the size fraction $0.125-2 \mathrm{~mm}$ reaching $5.2 \pm 0.3 \%$ at size fraction $0.71-1.2 \mathrm{~mm}$, with only $0.8 \%$ in the finer $(<0.125 \mathrm{~mm})$ and $0.02 \%$ in the coarser $(>2 \mathrm{~mm})$ fractions. Based on this analysis, in $3188 \mathrm{~g}$ of sieved WPCB (Table 1), there are approximately $18.8 \%$ of copper $(599.7 \mathrm{~g}), 3.4 \%$ of tin (107.2 g), and $1.3 \%$ of lead ( $42.6 \mathrm{~g})$ to be recovered. Consequently, the mass difference from the soluble fraction results in $27.5 \%$ of the initial material $(876 \mathrm{~g})$; according to EDS analysis (Figure 5), the presence of nickel, zinc, silicon, and aluminum was also identified in the samples.

SEM images of WPCB samples, shown in Figure 5, reveal that the geometrical properties of particles are very heterogeneous in terms of size, size distribution, and shape. The geometric properties of particles affect the particle flow behavior through an interaction with the gas medium as exhibited by the drag force, the distribution of the boundary layer on the particle surface, and the generation and dissipation of wake vortices ${ }^{25}$. In addition, the chemical composition of samples also changes considerably with size fraction, as verified in AA and EDS analysis, affecting the average density of particles. As the flow characteristics of solid particles in a gas-solid suspension varies with the geometric and material properties of the particles, and considering the extremely irregular shape of the particles of WPCB, a zigzag air classifier was chosen because its particular geometry subjects both streams of moving particles (the one carried upward by the up flowing air current and the one moving downward along the lower wall) to a renewed classification at the end of each stage.

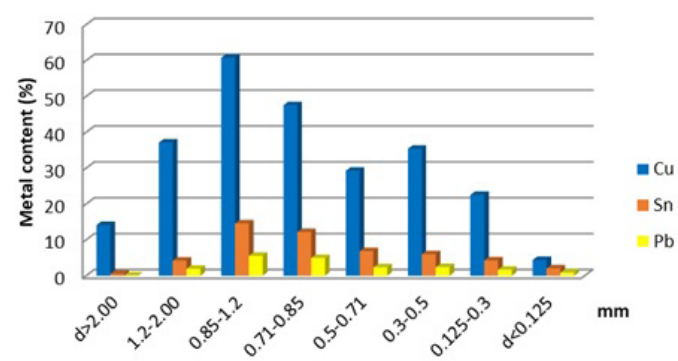

Figure 4. Metal distribution (copper, lead, and tin) in the soluble fractions with particle size fractions.

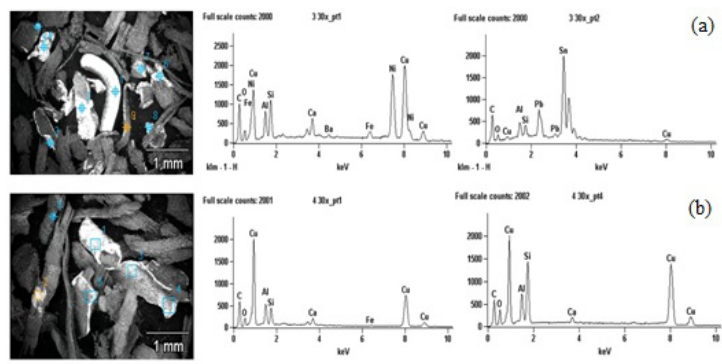

Figure 5. SEM images of samples (a) $0.3-0.5 \mathrm{~mm}$, and (b) $0.5-0.71 \mathrm{~mm}$.

\subsection{Zig-zag pneumatic separation step}

Based on the previous steps and aiming at designing a processing route to treat WPCB, the sample could be divided into the following three main size fractions: finer $(<0.3 \mathrm{~mm})$, intermediate $(0.3-2.0 \mathrm{~mm})$, and coarser $(>2.0 \mathrm{~mm})$. For the zig-zag separation step, solely the intermediate size fraction was chosen for investigation because it has approximately 
$2 / 3$ of the total weight and concentrates $90.7 \%$ of copper, $94.5 \%$ of tin, and $95.6 \%$ of lead present in the WPCB residue. The finer fraction $(<0.3 \mathrm{~mm}$, representing $15.9 \%$ of the total weight) and the coarser fraction $(>2.0 \mathrm{~mm}$, representing $21.5 \%$ of the total weight) contained mostly insoluble materials (67\% of this fraction weight consist of ceramics and polymers), with only $9.3 \%$ of copper, $5.5 \%$ of tin, and $4.4 \%$ of lead originally present in the sample.

Table 2 summarizes the calculated and the experimental flow rate of air used in the zig-zag air classifier for each particle size of the intermediate size fraction $(0.3-1.2 \mathrm{~mm})$, including their relative density. The decrease in the values of the relative density observed in the smaller fractions is directly related to its composition, since these fractions present high levels of ceramics and polymers, as shown in Figure 3.

Table 2. Air flow rate (calculated and experimental) used in the zig-zag air classifier for each particle size in the intermediate size fraction, including their relative density.

\begin{tabular}{lccc}
\hline $\begin{array}{l}\text { Size fraction } \\
(\mathrm{mm})\end{array}$ & $\begin{array}{c}\text { Density } \rho \\
\left(\mathrm{g} / \mathrm{cm}^{3}\right)\end{array}$ & \multicolumn{2}{c}{ Air flow rate $\left(\mathrm{m}^{3} / \mathrm{h}\right)$} \\
\hline $0.85-1.20$ & 3.6 & $21.9-296.6$ & 18.0 \\
$0.71-0.85$ & 3.2 & $17.9-246.7$ & 16.5 \\
$0.50-0.71$ & 2.4 & $11.4-163.5$ & 15.0 \\
$0.30-0.50$ & 2.3 & $5.8-113.7$ & 13.0 \\
\hline
\end{tabular}

The measured relative density of each size fraction was used to calculate the range of flow rate of air to fluidize the particles in the zig-zag air classifier using the Schytil's diagram, assuming spherical shape particles ${ }^{26}$. A typical diagram is shown in Figure 6. As expected, lower flow rates of air are required for fluidization when smaller particles are treated. In the range of air flow rate shown in Table 2 (fluidization region), the separation between light and heavy particles is expected to occur quickly, since the drag is almost instantaneous, less than 20 seconds. Below the calculated range (fixed bed region), all particles tend to flow downward in the equipment because the air flow is not enough to drag the light particles; alternatively, when higher flow rates of air are used (pneumatic transport region), all particles may be dragged upward by the flow of air, including the heavy particles. Therefore, in both cases, no gravity separation is expected to occur. The experimental flow rate of air differed a little from those calculated, as shown in Figure 5, because the particles shape is nonspherical and extremely irregular; the theoretical porosity was used in the calculations and this may also reveal some effect. In particular, considering the flatted needle shape of the particles, a smaller flow rate of air is expected to fluidize them in the zig-zag air classifier, when compared to the fully spherical particles. A similar result was obtained by Sagratzki ${ }^{27}$ using Schytil's diagram, whose deviations between theoretical and experimental air

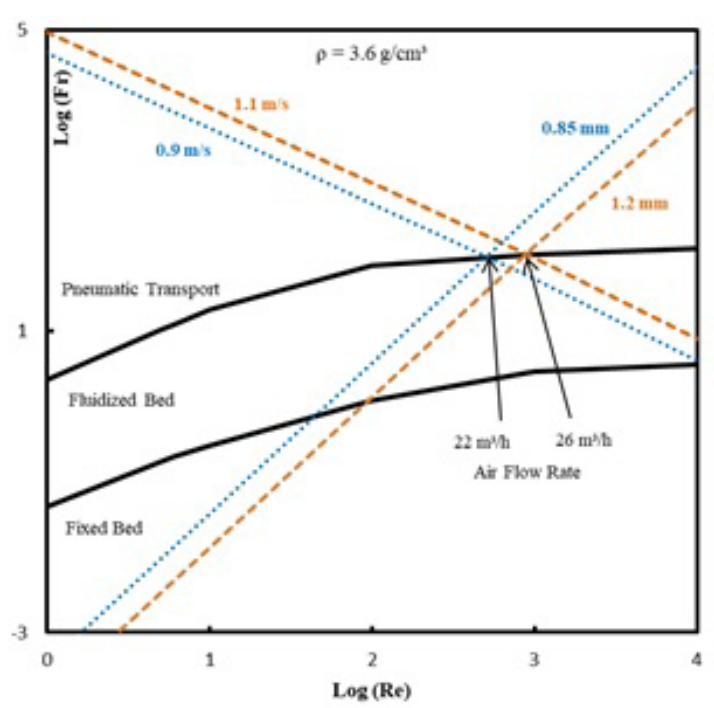

Figure 6. Schytil's diagram used to calculate the range of air flow rate for particle size range $0.85-1.2 \mathrm{~mm}\left(\right.$ density $\left.=3.6 \mathrm{~g} / \mathrm{cm}^{3}\right)$.

velocities were attributed to variations in porosity and shape of particles not considered in the calculations.

The total weight of light and heavy streams obtained in the zig-zag air classifier operated using the experimental air flow rate (given in Table 2) is shown in Figure 7. The light stream corresponds to the WPCB particles dragged upward with the air stream, which is mostly constituted by ceramic and polymeric materials. The heavy stream corresponds to the WPCB particles deposited in the collection box, which is mostly constituted by metals. The weight increase in the light stream (and consequent reduction on the weight of the heavy stream) when finer fractions of particles are treated fairly agree with the soluble (metal) and insoluble (ceramic and polymer) contents shown in Figure 3.

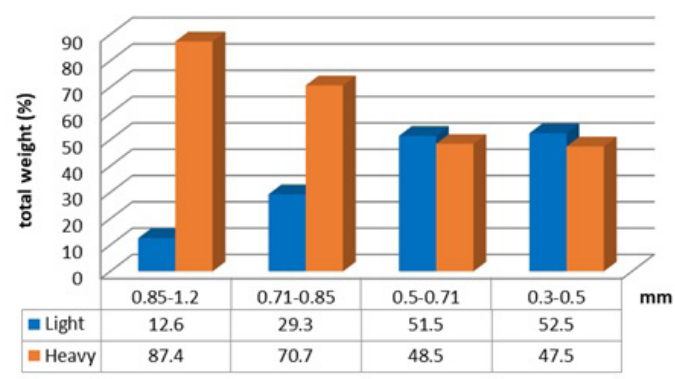

Figure 7. Total weight of light and heavy fractions after treatment of WPCB of distinct particle size distribution in the zig-zag air classifier.

The soluble fractions metals (copper, lead, and tin) content of both streams is shown in Figure 8. Metals were concentrated in the heavy streams as expected: in the size fraction $0.85-1.2 \mathrm{~mm}$, the content of copper increased from $60.7 \%$ to $72.5 \%$, tin from $14.5 \%$ to $18.4 \%$, and lead from $5.5 \%$ to $7.1 \%$; in the size fraction $0.71-0.85 \mathrm{~mm}$, the content 
of copper increased from $47.5 \%$ to $57.3 \%$, tin from $12.2 \%$ to $17.9 \%$, and lead from $4.9 \%$ to $6.6 \%$; in the size fraction $0.5-0.71 \mathrm{~mm}$, the content of copper increased from $29.2 \%$ to $72.2 \%$, tin from $6.8 \%$ to $14.6 \%$, and lead from $2.3 \%$ to $6.4 \%$; and in the size fraction $0.3-0.5 \mathrm{~mm}$, the content of copper increased from $35.4 \%$ to $69.2 \%$, tin from $6 \%$ to $15.7 \%$, and lead from $2.4 \%$ to $5.4 \%$. The heavy stream corresponds to $63.5 \%$ of total mass weight treated in the zig-zag air classifier, while the light stream corresponds to $36.5 \%$ in mass weight. The separation between soluble (metal) and insoluble (ceramic and polymer) materials was quite fast and efficient. In the light stream, $72.6 \%$ of the mass weight comprised insoluble materials, whereas $27.4 \%$ comprised soluble materials with low metal content of copper, tin, and lead. In the heavy stream, $9.8 \%$ of the mass weight comprised insoluble materials, whereas $90.2 \%$ of the mass weight comprised soluble materials, concentrated in copper, tin, and lead.

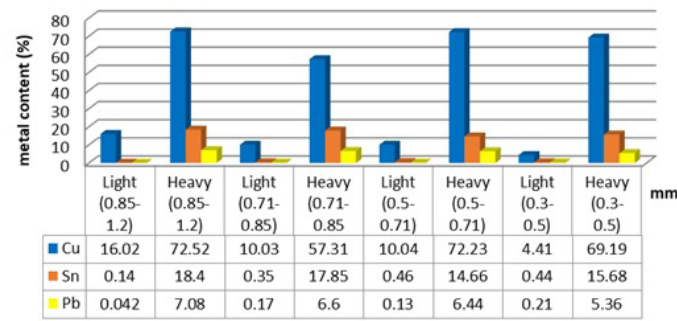

Figure 8. Metal distribution (copper, lead, and tin) in the soluble fractions of both streams for distinct particle size of zig-zag air classifier.

\section{Conclusions}

WPCB were mechanically treated (manual dismantling, comminution, magnetic separation, sieving, and gravity separation by air) aiming at separating the non-metallic fraction and to concentrate the metal content for subsequent recovery of copper, tin, and lead. The proposed route has generated no liquid or gaseous effluent to the environment.

The following conclusions can be drawn:

- Approximately $10.7 \%$ of the obsolete WPCB (excluding capacitors, resistors, CPUs, fan/cooler, heat-dissipator, soldered components and others containing precious metals like gold, silver and palladium that were manually dismantled) was comprised of magnetic materials;

- $\quad$ The milled WPCB evaluated in this work consisted of approximately $50 \%$ of soluble (metal) materials and $50 \%$ of insoluble (ceramic and polymer) materials. The metals content in the residue was $18.8 \%$ of copper, $3.4 \%$ of lead, and $1.3 \%$ of tin. Minor content of other metals like nickel, iron, zinc, and aluminum were also identified;
- Insoluble materials concentrated in the finer $(65 \%$ in $0.125-0.3 \mathrm{~mm}$ and $77 \%$ in $<0.125 \mathrm{~mm}$ ) and the coarser $(63 \%$ in $>2 \mathrm{~mm})$ size fractions of the residue. These fractions correspond to approximately one third of the total weight to be treated. In terms of metal content, these fractions contain $9.3 \%, 5.5 \%$ and $4.4 \%$ of total copper, tin, and lead contained in the milled WPCB, respectively;

- $\quad$ Soluble materials concentrated (from $47 \%$ to $74 \%$ ) in the intermediate size fraction (between 0.3-2 $\mathrm{mm}$ ), corresponding to approximately $2 / 3 \mathrm{rd}$ of the total weight to be treated, which contains $90.7 \%$, $94.5 \%$, and $95.6 \%$ of the total copper, tin, and lead contained in the milled WPCB, respectively;

- The fractions between $0.3-1.20 \mathrm{~mm}$ were submitted to gravity separation using a zig-zag air classifier, which ensured efficiency and celerity in this step. Considering only these size fractions, the content of copper increased from $43 \pm 11 \%$ to $68 \pm 5 \%$, tin increased from $10 \pm 3 \%$ to $17 \pm 1 \%$, and lead increased from $4 \pm 1 \%$ to $6.4 \pm 0.5 \%$;

- The zig-zag classifier has shown to be a sustainable and energy saving alternative for the concentration of metals from WPCB. It is easy to use, has no internal parts, fast, very economic and environmental friendly using air at room temperature.

\section{Acknowledgments}

Authors acknowledge Brazilian agencies CNPq (Conselho Nacional de Desenvolvimento Científico e Tecnológico), CAPES (Coordenação de Aperfeiçoamento de Pessoal de Nível Superior) and FAPERJ (Fundação de Amparo à Pesquisa do Estado do Rio de Janeiro) for financial support.

\section{References}

1. Rubin RS, Castro MAS, Brandão D, Schalch V, Ometto AR. Utilization of life cycle assessment methodology to compare two strategies for recovery of copper from printed circuit board scrap. Journal of Cleaner Production. 2014;64:297-305.

2. Ning C, Hadi P, Aghdam E, Zhu S, Hui DCW, Lin CSK, McKay G. Environmental emission analysis of a waste printed circuit board-derived adsorbent production process. Chemical Engineering Journal. 2017;326:594-602.

3. Zhou Y, Wu W, Qiu K. Recovery of materials from waste printed circuit boards by vacuum pyrolysis and vacuum centrifugal separation. Waste Management. 2010;30(11):2299-2304.

4. Dutta D, Panda R, Kumari A, Goel S, Jha MK. Sustainable recycling process recovery for metals recovery from used printed circuit boards (PCBs). Sustainable Materials and Technologies. 2018;17:e0066.

5. Sum EYL. The recovery of metals from electronic scrap. Journal of Metals. 1991;43(4):53-61. 
6. Goosey M, Kellner R. Recycling technologies for the treatment of end of life printed circuit boards (PCBs). Circuit World. 2003;29(3):33-37.

7. Veit HM, Bernardes AM, Ferreira JZ, Tenório JA, Malfatti CF. Recovery of copper from printed circuit boards scraps by mechanical processing and electrometallurgy. Journal of Hazardous Materials. 2006;137(3):1704-1709.

8. He W, Li G, Ma X, Wang H, Huang J, Xu M, Huang C. WEEE recovery strategies and the WEEE treatment status in China. Journal of Hazardous Materials. 2006;136(3):502-512.

9. Zheng Y, Shen Z, Cai C, Ma S, Xing Y. The reuse of nonmetals recycled from waste printed circuit boards as reinforcing fillers in the polypropylene composites. Journal of Hazardous Materials. 2009;163(2-3):600-606.

10. Guimarães YF, Santos ID, Dutra AJB. Direct recovery of copper from printed circuit boards (PCBs) powder concentrate by a simultaneous electroleaching-electrodeposition process. Hydrometallurgy. 2014;149:63-70.

11. Xie F, Cai T, Ma Y, Li H, Li C, Huang Z, Yuan G. Recovery of $\mathrm{Cu}$ and $\mathrm{Fe}$ from printed circuit board waste sludge by ultrasound: Evaluation of industrial application. Journal of Cleaner Production. 2009;17(16):1494-1498.

12. Veit HM, Diehl TR, Salami AP, Rodrigues JS, Bernardes AM, Tenório JA. Utilization of magnetic and electrostatic separation in the recycling of printed circuit boards scrap. Waste Management. 2005;25(1):67-74.

13. Li J, Xu Z, Zhou Y. Application of corona discharge and electrostatic force to separate metals and nonmetals from crushed particles of waste printed circuit boards. Journal of Electrostatics. 2007;65(4):233-238.

14. Duan C, Wen X, Shi C, Zhao Y, Wen B, He Y. Recovery of metals from waste printed circuit boards by a mechanical method using a water medium. Journal of Hazardous Materials. 2009; 166(1):478-82.

15. Zhou Y, Qiu K. A new technology for recycling materials from waste printed circuit boards. Journal of Hazardous Materials. 2010;175(1-3):823-8.

16. Gerbase AE, Oliveira CR. Reciclagem do lixo de informática: uma oportunidade para a química. Química Nova. 2012;35(7):14861492.
17. He J, Duan C. Recovery of metallic concentrations from waste printed circuit boards via reverse flotation. Waste Management. 2017;60:618-628.

18. Zhang G, Wang H, He Y, Yang X, Peng Z, Zhang T, Wang S. Triboelectric separation technology for removing inorganics from non-metallic fraction of waste printed circuit boards: influence of size fraction and process optimization. Waste Management. 2017;60:42-49.

19. Xia MC, Wang YP, Peng TJ, Shen L, Yu RL, Liu YD, et al. Recycling of metals from pretreated waste printed circuit boards effectively in stirred tank reactor by a moderately thermophilic culture. Journal of Bioscience and Bioengineering. 2017;123(6):714-721.

20. Zhang G, He Y, Wang H, Zang T, Wang S, Yang X, Xia W. New technology for recovery residual metals from nonmetallic fractions of waste printed circuit boards. Waste Management. 2017;64:228-235.

21. Park YJ, Fray DJ. Recovery of high purity precious metals from printed circuit boards. Journal of Hazardous Materials. 2009;164(2-3):1152-8.

22. Pacheco AMV. Eletrorrecuperação de zinco sobre catodo fluidizado numa célula cônica [dissertation]. Rio de Janeiro (RJ): Federal University of Rio de Janeiro; 1981.

23. Szekely J, Thermelis NJ. Rate phenomena in process metallurgy. New York: John Wiley \& Sons; 1971.

24. Silvas FPC, Correa MMJ, Caldas MPK, Moraes VT, Espinosa DCR, Tenório JAS. Printed circuit board recycling: physical processing and copper extraction by selective leaching. Waste Management. 2015;46:503-510.

25. Fan LS, Zhu C. Principles of gas-solid flows. Cambridge: Cambridge University Press; 1998.

26. Ribeiro PPM, Santos ID, Dutra AJB. Copper and metals concentration from printed circuit boards using zig-zag classifier. Journal of Materials Research and Technology. 2019;8(1):513520.

27. Sagratzki NL. Parâmetros de fluidização do minério de cobre de caraibas [dissertation]. Rio de Janeiro (RJ): Universidade Pontífica Católica; 1975. 